\title{
PROCREATION AND APPLICATION OF SENTIMENT BASED DICTIONARY TO REVEAL POPULAR EVENT TOPICS TRENDS ON TWITTER PLATFORM
}

\author{
Poonam Vijay Tijare \\ Research Scholar, Department of Computer Science and Engineering (VTU RC), \\ CMR Institute of Technology, Bangaluru, Karnataka, India-560037 \\ poonamtijare@gmail.com \\ Jhansi Rani P. \\ Professor, Department of Computer Science and Engineering (VTU RC), \\ CMR Institute of Technology, Bangaluru, Karnataka, India-560037 \\ jhansirani.p@gmail.com
}

Abstract

The layers of information are formed by the rapid rise of the data storm that is generated on Social Media(SM). The work of finding hidden trends is tough owing to the constant flow of tweets caused by volume and variety. The study recommends the use of dictionaries to absorb information more quickly and filter out inadequate data.

The research proposes the procedural method to build the global dictionary. The global dictionary is created using the datasets on social, political, scientific and sports domains on most recent events to cover major sentiment drifts. This dictionary can help in revealing trends which can further be used to detect the event trends.

The suggested model performs trend analysis on a tweet-by-tweet basis using dictionaries. A tweet-specific trend evolves into a day trend for an event. The proposed model aids in the examination of trends for an event on a timeline. The event trend has the ability to reveal the event's future trajectory. The model is validated using a multi-event dataset from Event 2012 and evaluated successfully on a Citizenship Amendment Act (CAA) dataset from 2019. The model performs well across a wide range of datasets.

Keywords: Social Media; Twitter; Dictionary; Event; Topic; Trends.

\section{Introduction}

The Proliferous amount of data getting generated at every second on social media raises the curiosity to analyse its relation and reflection on societal behaviour. Twitter is the most commonly and widely used platform by the public to outcast their opinion and views on various topics. With $500 \mathrm{M}$ tweets each day and 6000 TPS (tweets per second) makes Twitter a popular platform on SM. Twitter experienced a spike of 143199 tweets per second (TPS) on August 2 and 3, 2013 for an air show event in Japan ${ }^{1}$. It reflects that the important events bring out the emotions of the people. SM had given the voice to the people across continents to pen down their opinions fearlessly without any pressure. Mining down this information repository can reveal many things on likings, culture, lifestyle, products and so on. This made Twitter data analytics the most sought after research area. The researchers are performing various types of analytics to reveal the important details from this information depository.

One of the important applications of Twitter data mining is trending event identification involving detection and prediction of popular events. Twitter data is huge and diverse with short tweets. Extracting overriding patterns continuously getting generated is an upcoming challenge. To deal with the information at a faster pace, the high computation algorithms are required [Zhou (2020)]. Continuous addition of information may lead to delay or give the misleading analysis on data. Processing of irrelevant information refrains the system performance. The state of the art problem demands the need of well-designed filters which can sieve irrelevant information. Building a global dictionary covering a variety of events can be a solution for this problem. This will help filtering tweets in a systematic manner leading to faster processing [Chen (2019)][ Saeed (2019)].

However, the bigger the dictionary size, the more time system will take to scan and apply. Hence, it is of utmost importance to keep the size of the dictionary as small as possible with full coverage of inevitable information. This research paper proposes a novel method to create the compact sentiment driven dictionary to

\footnotetext{
${ }^{1}$ https://blog.Twitter.com/engineering/en_us/a/2013/new-tweets-per-second-record-and-how.html
} 
reveal events. The sentiment driven dictionaries are created using diverse local and global events happening in 2019. Dictionaries are then applied to the event detection model. The benchmark datasets Event 2012 and Citizenship Amendment Act(CAA) 2019 are used to test the global dictionary. The work is the extension of the method proposed in [Tijare (2021)].

Contributions of paper can be summarized as,

(1) Algorithm to get a combined dictionary from diverse events.

(2) The dictionary filtering algorithm to produce the compact dictionary based on frequency and sentiment of each word.

(3) The algorithm on dictionary application on benchmark datasets.

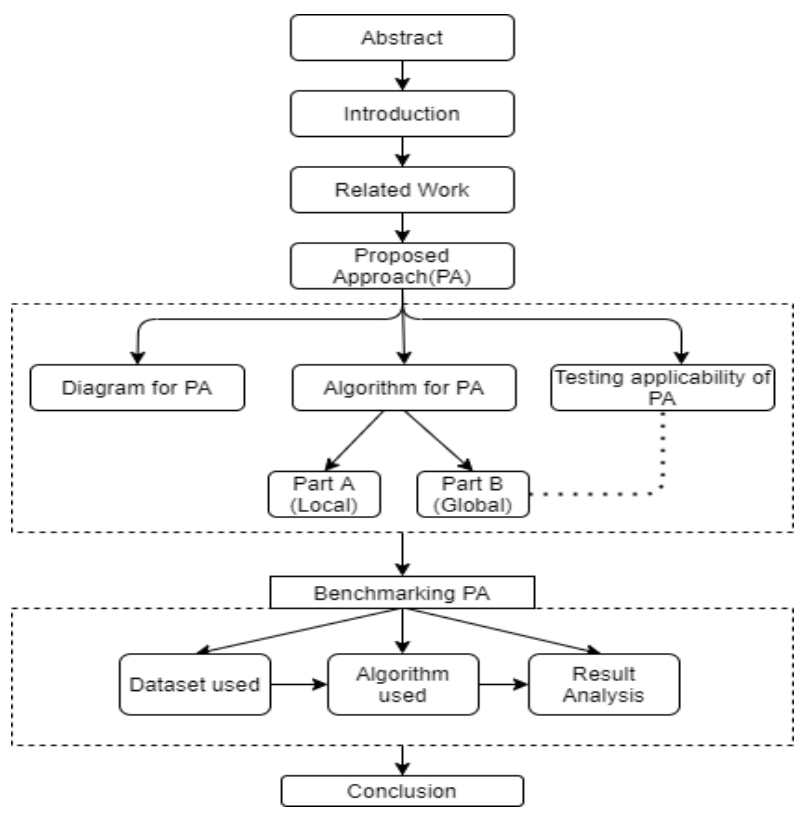

Fig 1. Organisation of paper

The Figure 1 shows the organization of the research paper. The overview of the present approaches is highlighted in section 2. Subsequent sections present the steps to build the dictionary, and the application of sentiment driven dictionary on benchmark datasets.

\section{Related Work}

Most of the methods proposed by researchers are using topic modelling methods to find micro topics. They use an unsupervised learning approach where the total numbers of tweets are divided into a number of topics based on similarity and word frequency. Total number of topics can be decided by training a model with combinations of varying topic numbers and log likelihood scores. Apart from that, deciding total topics prior is not ideal in all situations. Topic modelling algorithms requires considerable amount of time, hence cannot be preferred choice for streaming data analysis [Tijare (2020)][Nolasco (2019)].

Irrelevant, missing or incomplete data brings down the performance of a model. Analysts had pointed the utmost need of filtering methods to remove unwanted tweets [Hasan (2019)]. Researchers used neural networks on data from Instagram and Twitter for event prediction [Mukhina (2019)]. Linmei Hu et al. [Hu (2020)] proposed the hierarchical attention based model for event identification. The model learns with word sequences with marginal accuracy. Yang et al. [Yang (2019)] used Flickr for event identification. Model learns through a dictionary on differing events from diverse domains, gives better accuracy with geospatial data. Alomari et al. [Alomari (2020)] used dictionaries to label the traffic events as relevant and not relevant. Dictionaries contain the Arabic words with the total occurrence count. Models use a TF/IDF (Term Frequency/Inverse Document Frequency) based feature extractor and specially designed stemmer. Proposed model is tested for traffic events.

Wilson et al. [Wilson (2020)] presented the correlation analysis between use of words on a platform called Urban dictionary and Twitter trending events. It is a crowdsourced platform presenting the short terms used for phrases that are used on SM platforms. Jeffery Ansah et al. [Ansah (2020)] presents the SensorTree model using network connections amongst the user. Model uses a dictionary of protest related terms to filter out tweets containing protest data. Model also uses the LDA (Latent Dirichlet allocation), frequent keywords with 
EventTweet framework for detecting events related to protests. Kilicoglu and Bergler created the heuristic rule based dictionary on biological events and mentioned that these methods are suitable for noisy datasets [Kilicoglu and Bergler (2009)].

Dictionaries created so far are restricted to filter out irrelevant tweets. They can be utilised in a better way to perform the analysis in much detail on tweet level. The research proposes the approach for creating dictionaries by taking reference of miscellaneous dominions. The dataset belongs to dominions like -

Sports - Cricket World Cup 2019,

Political - Deal on Rafale Jets used in Election 2019 India,

Scientific - Launch and testing of Mission Shakti project, and

Social - Sabarimala temple event on banning female devotees inside temple premises.

\section{Proposed Approach}

The research work focuses on generation of dictionaries. At the initial level, the dictionary for individual dataset is created and then all the individual dictionaries collectively form a combined dictionary. Further, the global dictionary goes through the filtration process resulting into a compressed dictionary. These dictionaries are equipped with,

(1) Collection of most influential nouns.

(2) Nouns are presented with the overall frequency and sentiment of the context in which it is used in tweets across all the datasets used to form a global dictionary.

The global dictionary is created using datasets gathered in 2019. The datasets are formed using Twitter API Tweepy for the stipulated timeline of various events. Dictionary formation is performed using datasets shown in the figure 2. The timeline of tweets and number of days for which tweets are extracted from Twitter using keyword based search are presented in figure 2 .

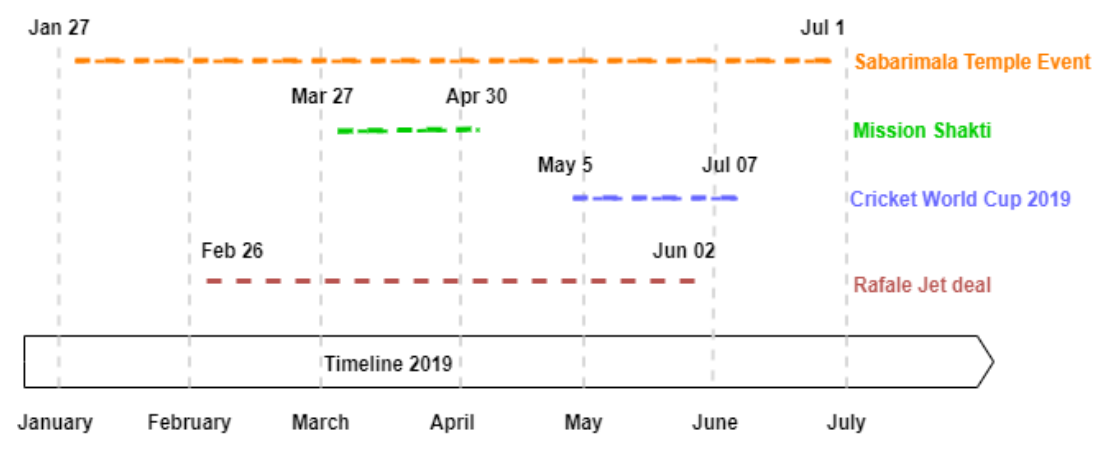

Fig 2. Timeline of tweets extracted for events

\subsection{Dictionary formation algorithm}

As discussed in section 1, the dictionary created should be compact and must provide faster access to data. The dictionary creation is a two phase process,

Part A: Generation of individual dictionary for each dataset.

Part $B$ : The formation of global dictionary and further optimization.

All datasets considered in formation of dictionary collectively size up to 382,194 relevant tweets. Figure 3 shows the flow diagram for dictionary creation algorithms in Part A and Part B. The left side of the diagram depicts the formation of dictionary for individual dataset. These dictionaries are then collectively forms the global dictionary. The process of generating this global dictionary is showcased in the right side of the diagram. The individual steps are briefed in the following algorithms (Part A and Part B) and elaborated in later sections.

Part A: algorithm_dictionary_generation

Initialize dataset $D=$ [Rafel Jet deal, Sabarimala, Mission Shakti, World cup 2019]

for each dataset in $D$ :

Tweet [ $=$ Combine day-wise tweets

for each tweet in tweet[]

Nouns[]= Nouns in tweet

for each word in Noun []:

calculate sentiment

calculate frequency of words 
find Sentiment of the tweet containing this word find Subjectivity of the tweet containing this word

The above algorithm generates dictionaries for each dataset mentioned in figure 2 and returns the individual dictionary for all datasets. The Enchant English dictionary API for Python 3.0 is used to find the Nouns. VADER (Valence Aware Dictionary for Sentiment Reasoning) and TextBlob sentiment analysers are used to find sentiments. TextBlob uses SentiWordnet with Pattern library for polarity of words and adjective dictionary with scores. In contrast, VADER performs the sentiment analysis considering polarity as well as emotion aspect on sentiment by forming lexical dictionaries and hence VADER is favourable option over TextBlob. TextBlob is used to find the subjectivity of the tweets with shortlisted nouns.
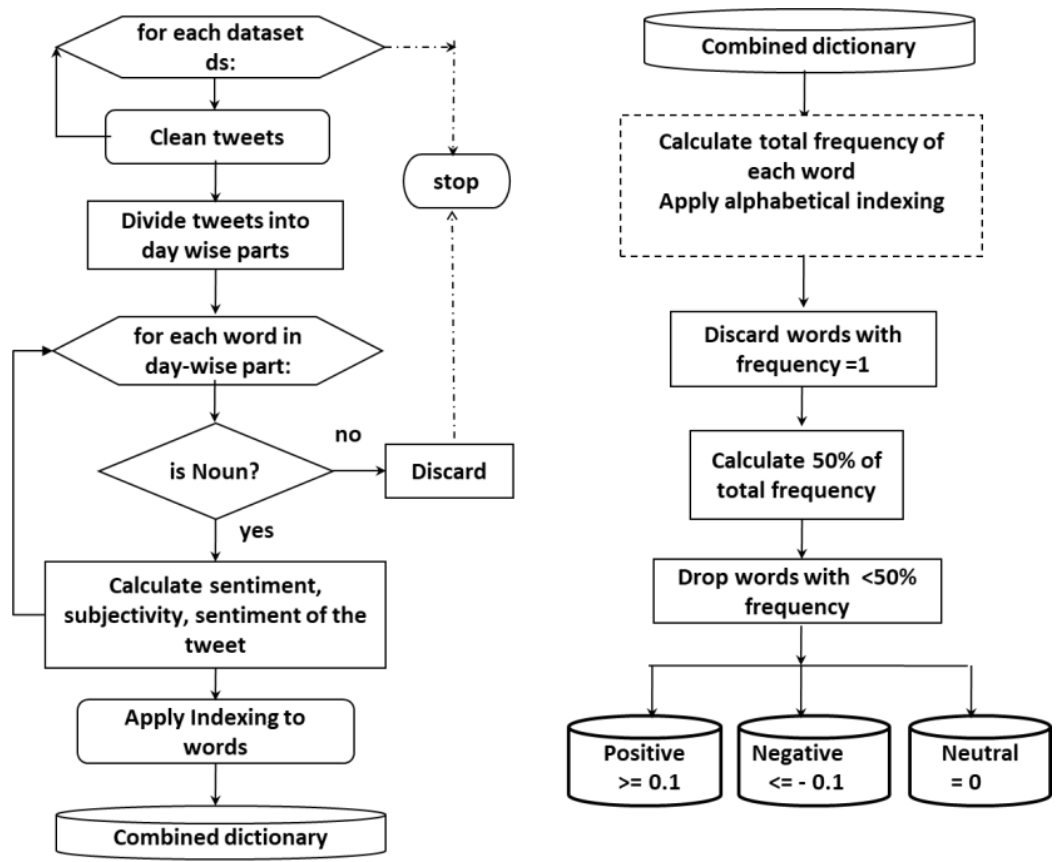

Fig 3. Flow diagram for dictionary creation algorithms in Part A and Part B

The size of the dictionary built using Part A algorithm resulted into 160891 words. Processing this dictionary resulted in slow response time and highlights the necessity of compressing the dictionary. Part B algorithm compresses the dictionary by adding filters to form a compact dictionary.

Part B: Algorithm on dictionary optimization dictionary[] = Combine all dictionaries [Rafale deal, World Cup, Mission Shakti and Sabarimala] for each word in dictionary []:

Calculate total frequency, maximum and average sentiment

Delete the words with frequencies=1 from dictionary[ ]

Apply alphabetical sorting to dictionary words

Build indices for all the alphabets

Calculate 25, 50 and 75 percentiles.

Set threshold $=50 \%$ percentile

for each word in dictionary[]:

if word < threshold:

Discard word from dictionary[]

Initialize :

Positive word dictionary[]

Negative word dictionary[]

Neutral word dictionary[]

for each word in dictionary[]:

If sentiment > 0.01 :

add word to Positive word dictionary[]

If sentiment $<-0.01$ : 
add word to Negative word dictionary[]

If sentiment $=0$ :

add word to Neutral word dictionary[]

The part B algorithm forms three dictionaries according to their sentiment values. The algorithm considers individual dictionaries generated by part A. All these dictionaries are combined to form one global dictionary. All the words in this global dictionary are then processed to compute the total frequency, sentiment and subjectivity scores. All the words with frequency one are discarded as they were having the least overall impact across all datasets.

The distributions of words in the global dictionary are observed using box plots. The percentile scores are taken to understand the occurrence frequency of the words. The words with frequency less than 50 percentiles are discarded as they are irrelevant in the process of detecting an event. The figure 4 (a) and (b) shows the graphs showing the distribution of words with respect to their frequencies. The figure 4 (b) shows the most of the frequencies falling in the range between 1000 and 4000 .

Next, by taking the starting index of words beginning from each alphabet are stored in the list to enable faster search. This indexing results in a remarkable decrease in search time. Figure 5 shows the time taken to search words in seconds without indexing and with indexing. To achieve the maximum coverage of search action over dictionary, the words scattered across the overall dictionary are chosen. The words 'abort' at the start, 'monk' in the middle, and 'zaheer' at the conclusion of the dictionary are considered to showcase the results. The average improvement in response time after considering the indexing is $87.01 \%$.
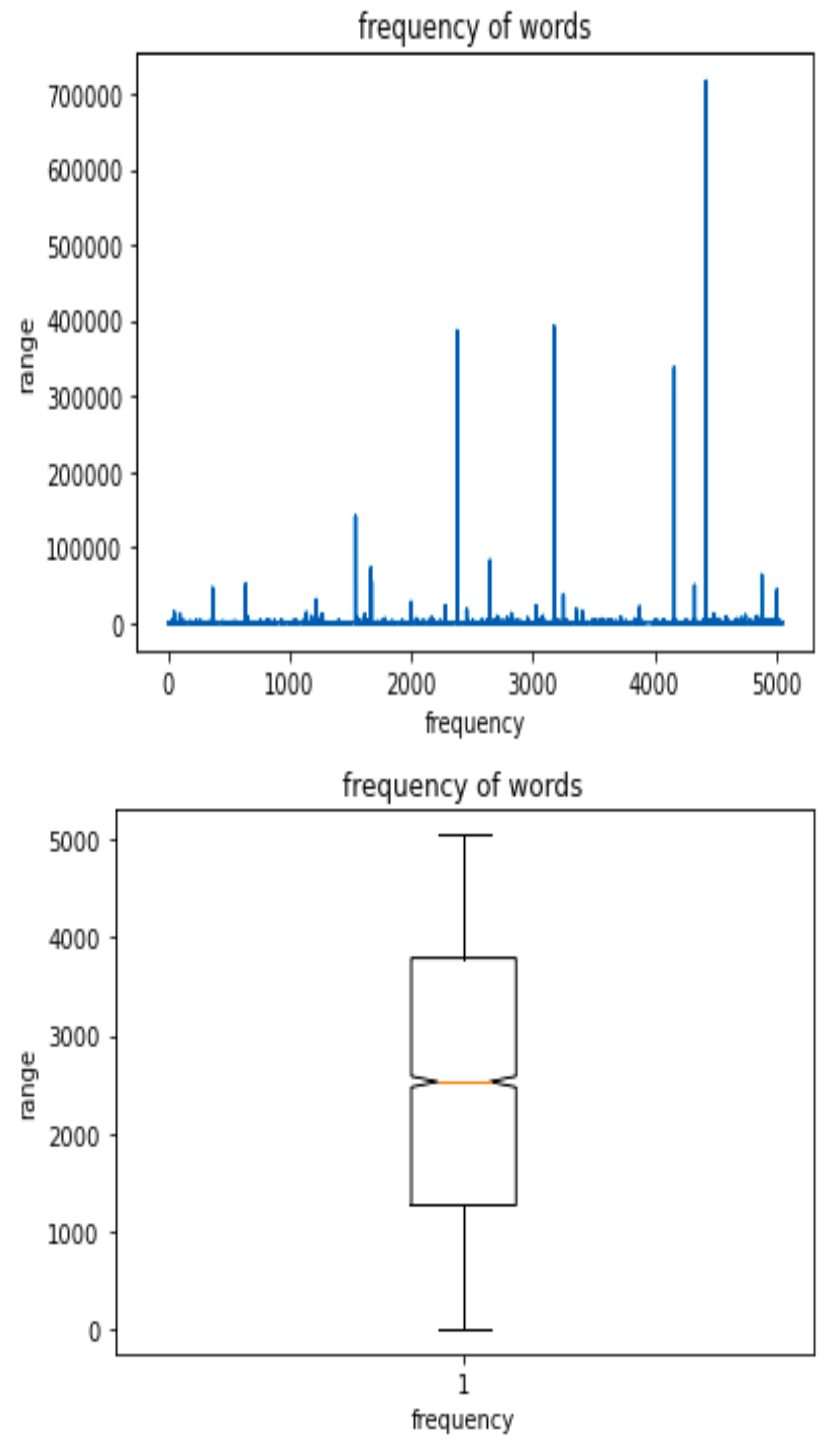

Fig 4. a) Frequency of all Nouns b) Overall weight of frequencies 


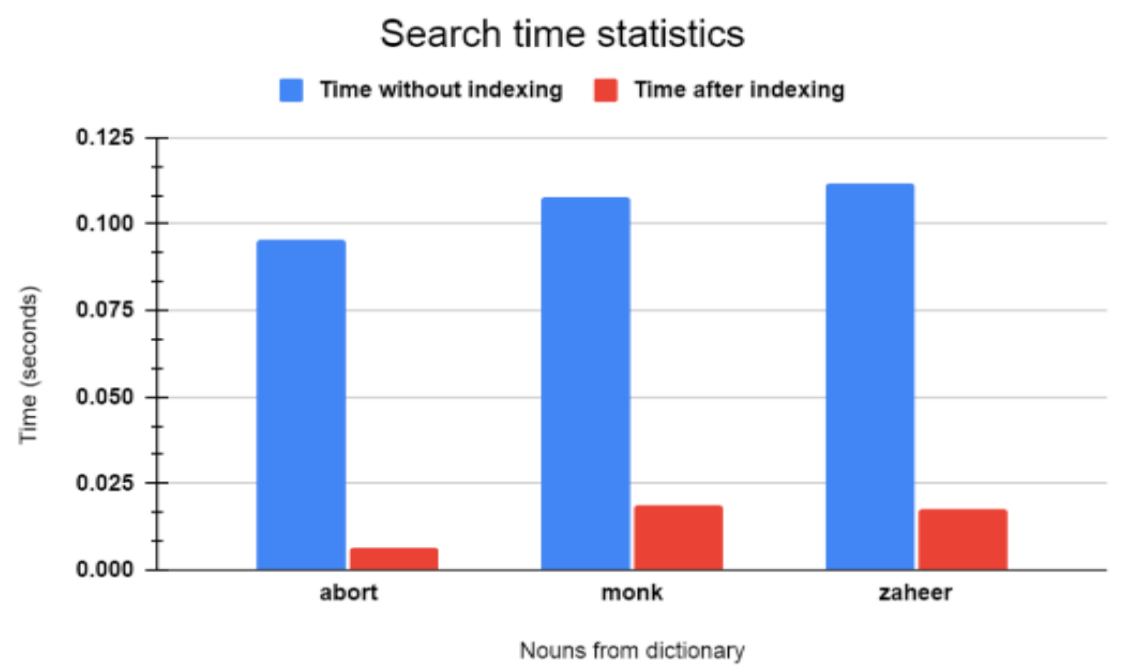

Fig 5. Search time statistics for the words in dictionary

The original size of the dictionary is of 160981 words. The drastic compression of the dictionary with 2569 words is achieved by applying the indexing method. This approach considers the percentile threshold value to discard irrelevant words. In the concluding phase, the global dictionary is divided into three sub-dictionaries as positive, negative and neutral word dictionaries. The threshold value is set by taking reference of distribution of sentiment score as shown in figure 6. It is observed from figure 6 that the words are clustered near -0.01 to 0.01 . Therefore, a positive word dictionary is formed by taking a sentiment threshold value greater than 0.01 , negative word dictionary formed with sentiment threshold less than -0.01 and the neutral word dictionary formed using sentiment value 0 . The purpose of forming positive dictionary, negative dictionary and neutral dictionary is to achieve better performance in searching.

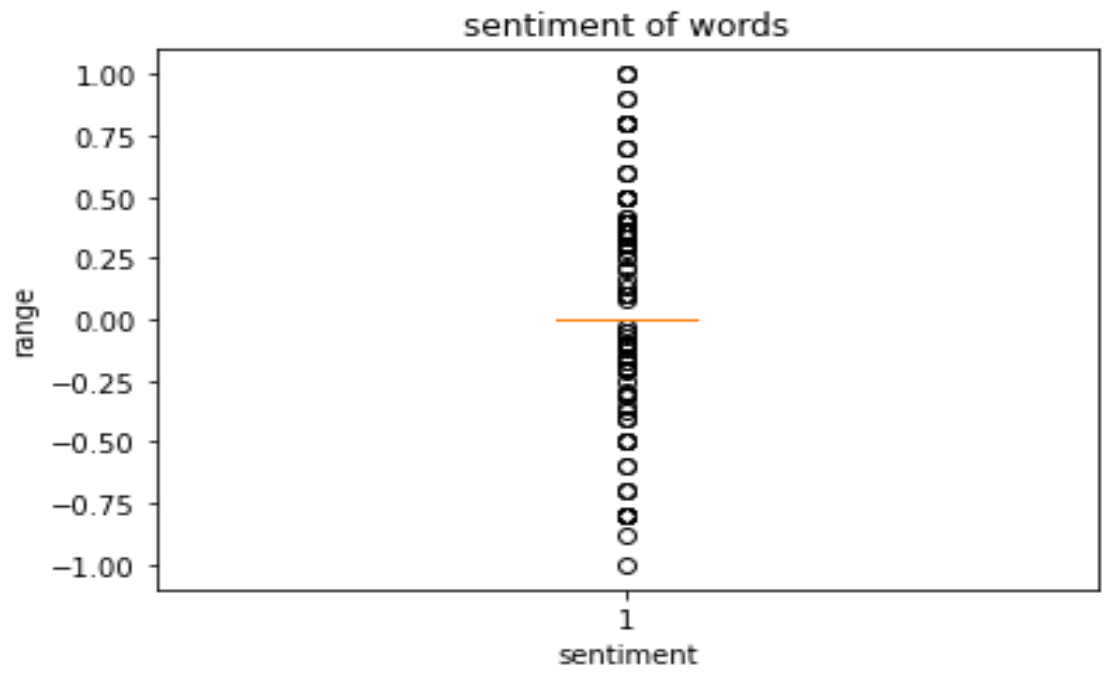

Fig 6. Sentiments of Nouns

Figure 7 shows the step by step reduction in the size of the dictionary after application of each filter discussed in Part A and Part B algorithm. The application of algorithms resulted in a compact dictionary with compression rate $96.89 \%$. 


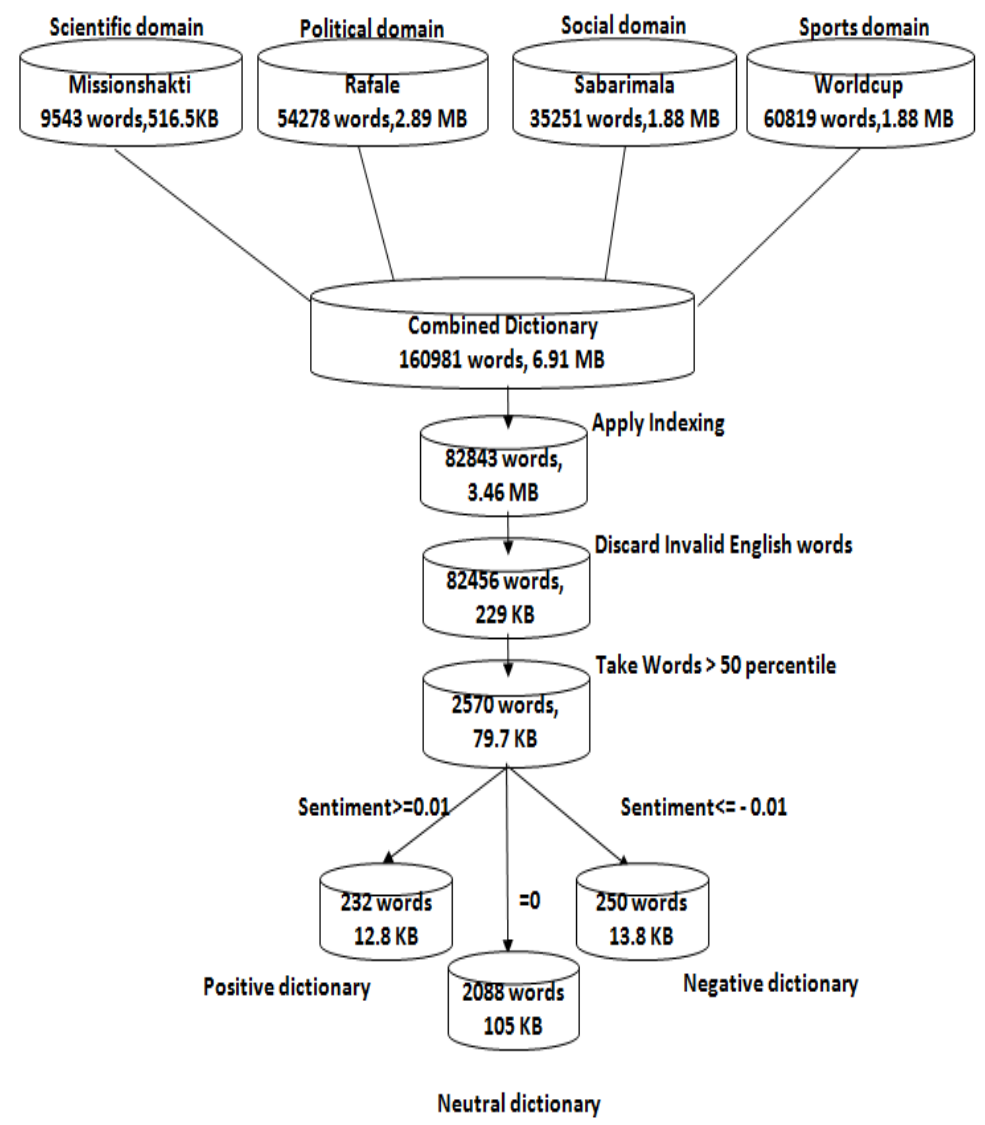

Fig 7. Flow of dictionary creation with size

\section{Validation and Application of the Dictionary}

The global dictionary is generated based on the recent events that happened in 2019. It is utmost important to expose model to diverse data to test the applicability of the dictionary. The Event 2012 [Papadopoulos (2013)] and CAA [Cherian (2019)] datasets are considered. The trend detection algorithm using dictionary based approach is proposed in coming section.

\subsection{Trend detection procedure using Dictionaries}

The trend detection procedure presented ahead can reveal trends by performing daily tweet trend analysis. This algorithm is applied on Event 2012 and CAA datasets. The trend detection procedure can be applied to diverse events with variable timelines. The approach performs tweet wise analysis to decide the trends which eventually leads to events.

Proposed procedural approach for trend detection:

step 1 :

calculate $p 1=$ percentile (word_freq, 25)

p2 = percentile (word_freq, 50)

p3=percentile (word_freq, 75 )

where, word_freq is frequency of each word in combined dictionary formed in step 5 of PART B

step 2:

for each tweet $t$ :

calculate pos-word $=t$ [words] $\cap$ Positive word dictionary

$n e$-word $=t$ [words] $\cap$ Negative word dictionary

$z$-word $=t$ [words] $\cap$ Neutral word dictionary

where, pos-word - collection of positive words,

neg-word - collection of negative words,

$z$-word - collection of neutral words

Find tweet $[$ trend $]=\max ($ pos_word, neg-word, $z$-word $)$

Label tweet [trend] $=t$

where, $t$ is -1 if trend is negative 


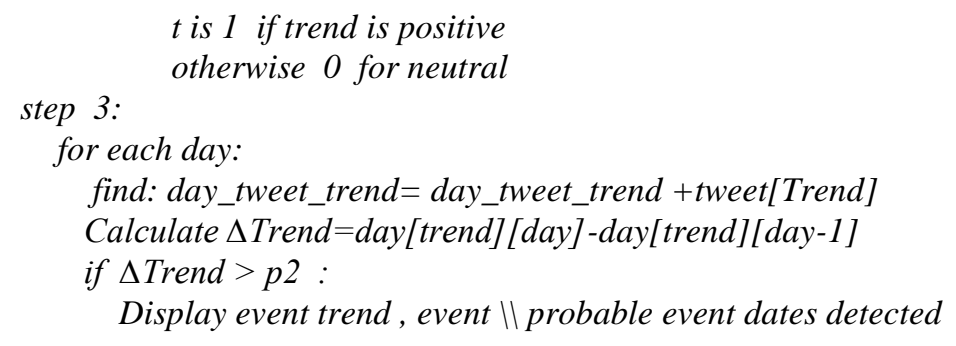

Initially the percentile scores are computed as shown in step 1 of the procedure. These scores help to reveal the percentage of tweets coming under $25 \%, 50 \%$ and $75 \%$ category. $50 \%$ score is taken as the threshold value to decide the trending event as this threshold value was giving good coverage of events.

For each tweet, compute the positive (pos-word), negative (neg-word) and neutral (z-word) words by referring to three dictionaries created by applying the algorithm discussed in part B. Trend will be decided considering the maximum count of the pos-word, neg-word and $\mathrm{z}$-word. The trends are set by labelling 1 for positive, 0 for neutral and -1 for negative. The trends labelling should be done for the tweets of each day. It is very important to understand how the event flows on the timeline. If the event flows continuously towards a negative direction, it reflects the alarming situation. Hence, it is very crucial to find the difference in trends by taking the difference between previous day and current day trend [Tijare (2021)]. If the change in trend is more than 50 percentile value computed on frequent words stored in a global dictionary, leading to the possible event based on the trend pattern. The algorithm detects the trend whenever significant events happen.

\subsection{Testing dictionary with Event 2012}

The Event 2012 dataset touches various social events contributing to validate the generated dictionaries. The Event 2012 dataset discusses various events by event-id. The event-id is further tagged with event description. Part of this dataset is public. The tweet for a given tweet-id of labelled events are downloaded using Twitter API. The Tweets are then cleaned and tokenised. The sentiments of the tweets are found using VADER sentiment analysis. Tweets are labelled for associated trend as discussed in previous section to compute $\Delta$ Trend. Dataset tweets are labelled for events as: if the $\Delta$ Trend is more than the set percentile score then positive, less than 0 then negative and neutral if equal to 0 .

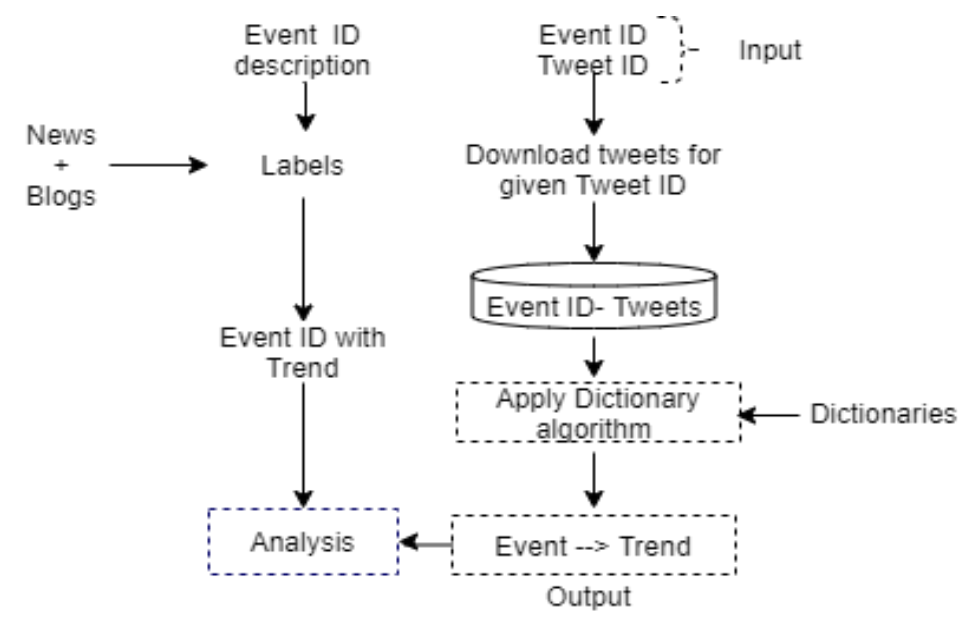

Fig 8. Dictionary application activity on Event 2012

Figure 8 depicts the activity to detect trend on Event 2012 dataset. Each Event ID associated with an event is labelled with trend. Table 1 shows the Even 2012 events with the mapped events and the trend labelled as positive, negative and neutral. Events with id 129, 257 and 131 are moving with a neutral trend in the timeline. The proposed Dictionary algorithm is applied on the dataset, resulting into Event and associated trend. The trend labels shown in table 1 are matching with the trends predicted by the model shown in detected trend column.

The proposed model yielded results with the least amount of human preprocessing. The Event 2012 dataset used for testing comprises 9967 events in total and the proposed dictionary algorithm detects 7076 events without any manual intervention in the labelling. The work is tested successfully with $71.91 \%$ accuracy. 
Table 1. Events with actual trends and detected trends

\begin{tabular}{|c|c|c|c|}
\hline Event ID & Events & $\begin{array}{l}\text { Labelled } \\
\text { Trend }\end{array}$ & $\begin{array}{l}\text { Detected } \\
\text { Trend }\end{array}$ \\
\hline 129 & Lenovo takes over HP as the biggest PC maker & Neutral & Neutral \\
\hline 257 & HP and Lenovo battle for top spot in PC marke... & Neutral & Neutral \\
\hline 131 & ABOUT LENOVO AND HP & Neutral & Neutral \\
\hline
\end{tabular}

(a) Daily trend analysis on CAA act

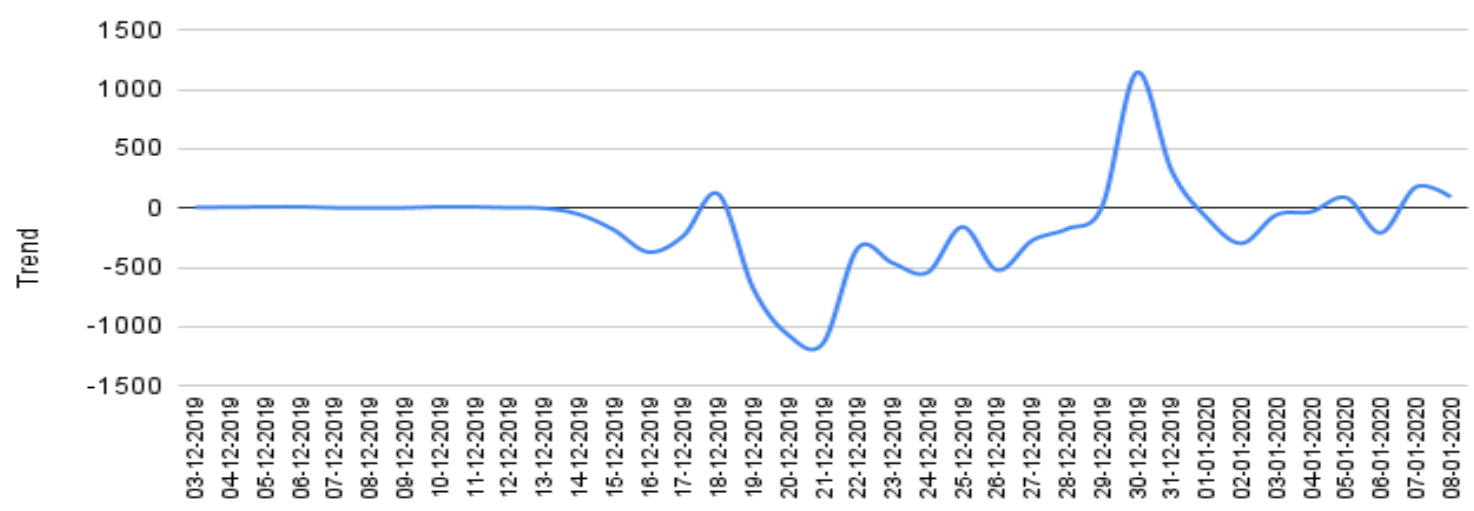

Date
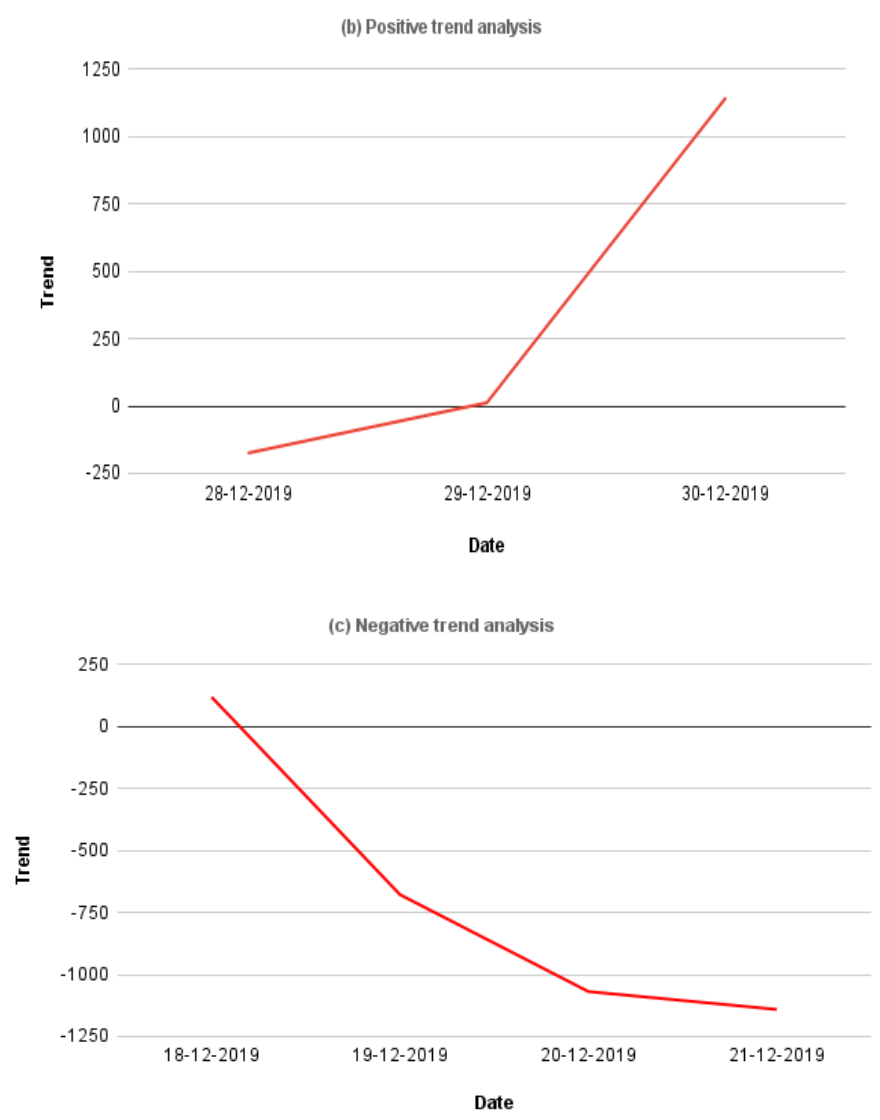

Fig 9.a) Trend movement on timeline b) Positive trend analysis c) Negative trend analysis 


\subsection{Application of Dictionary to reveal Trend on CAA 2019}

The CAA events began on the 9th of December 2019, when the law was introduced in the Indian parliament and passed in the following days. Protests erupted across India within days of the passing of the CAA bill. Many people were killed and property was damaged as a result of these events turning violent. The Shaheen Bag protest on CAA became increasingly polarized on social media and other outlets, sparking communal violence [Wikipedia contributors (2021)]. The entire episode was horrible and heart breaking. The suggested model's tweet sentiment analysis reveals trends and drift patterns that are linked to real-time occurrences.

The CAA dataset from Kaggle site has tweets extracted till January 09, 2020[Cherian (2019)]. Tweets and accompanying data are considered for experimental study from December 2019 to January 9, 2020. The features considered from dataset are date, time, tweet, place and hashtags. Tweets are preprocessed and vectorised. The scores for sentiment and subjectivity have been discovered. The proposed dictionary based procedure discussed in section 3.3.1 is applied on the CAA dataset. The model has successfully detected trends by performing tweet by tweet analysis. The trends detected by applying sentiment driven dictionary are shown from December 1 , 2019 to January 8th, 2020 in figure 9 (a). The movement in the trends can be observed after the CAA bill is enacted in the parliament on or after 11th of December 2019.

The trendline of the CAA event can be matched to real-time incidents that occurred during the event. The suggested model's detected trends can be used to determine the sensitivity of this event. The trend shifted from 28th of December towards positive direction as shown in figure 9(b). It was at its peak on December 30th. On December 29th, the Assam province minister and activist in Mumbai took part in a number of pro-CAA rallies. In the radio show "Mann ki Baat," the Prime Minister of India addressed the country amid CAA and NRC (National register of citizen) bill [THE QUINT (2019)]. The sentiment trend altered in a positive direction as a result of these events.

Figure 9(c) depicts the trend movement in the severe negative direction, suggesting a shift in the trend from 119 to -1141 from the 18 to the 21 of December 2019. The Supreme Court of India held its first hearing on CAA petitions on December 18, 2019[Sharma (2019)]. Protests upraised across the country and left-wing parties' consistent radicalization of events. These circumstances resulted into the deaths of innocent people during these violent protests. CAA was implemented on December 20th by the Union minister. On December 21st, the Prime Minister addressed the nation once more, urging people to keep the situation under control and to limit the execution of the CAA in Assam province. Figures 9(b) and 9(c) show how the positive and negative trend analysis validates the trends observed by the proposed model with real-time occurrences on CAA. The trend variations in figure 9(a) correspond to real-time incidents that occurred under the CAA statute.

The proposed model on sentiment driven dictionary can give greater accuracy with manual preprocessing. The model can be applied to any dataset, regardless of its domain or time period, to discover trending occurrences.

\section{Conclusion}

The proposed research work highlights the importance of identifying events and its societal impact. The work focuses on building the global dictionary for event detection by considering datasets in various domains. To achieve higher response time, the global dictionary is filtered and composed with $96.89 \%$ of compression rate. The indexing method is used to achieve quick response time with the improvement by $87 \%$. The global dictionary is then distributed in three individual dictionaries focusing on positive, negative, and neutral words. The dictionary is validated on Event 2012 dataset and applied on CAA 2019. The proposed algorithm detects the event with $71 \%$ of accuracy on Event 2012. The approach can detect a number of real-time events that occurred throughout the CAA 2019 period. The trend detection algorithm has the ability to recognise trends, and trend analytics can assist in forecasting future trends for a particular event.

The contributions of the suggested sentiment driven dictionary and trend identification approach can be summarised as follows:

- Compact sentiment driven dictionary.

- The detection of tweet-specific trends.

- Trend detection for a day.

- Using trend detection to gain a better picture of what's going on with certain events.

- Applicable on variety of datasets with varying timeline related to an event.

The work has considerable scope to extend the application of a dictionary on tweets to detect real time events on stream of tweets and their future developments. 


\section{References}

[1] Han Zhou, Hongpeng Yin, Hengyi Zheng, and Yanxia Li (2020). A survey on multi-modal social event detection. Knowledge-Based Systems, pp. 105-695.

[2] Xieling Chen, Shan Wang, Yong Tang, and Tianyong Hao (2019). A bibliometric analysis of event detection in social media. Online Information Review.

[3] Zafar Saeed, Rabeeh Ayaz Abbasi, Onaiza Maqbool, Abida Sadaf, Imran Razzak, Ali Daud, Naif Radi Aljohani, and Guandong Xu (2019). What's happening around the world? a survey and framework on event detection techniques on twitter. Journal of Grid Computing, 17(2), pp. 279-312.

[4] Poonam Tijare, Jhansi Rani P. (2021). Detecting Trending Event Topics Using Sentiment Driven Derivatives Method On Twitter. Vol. 12 No. 4 Jul-Aug 2021, pp 818-826.

[5] Tijare P. and Rani P.J (2020). Exploring popular topic models. In Journal of Physics: Conference Series, Vol. 1706, No. 1, pp. 012171. IOP Publishing, December.

[6] Diogo Nolasco and Jonice Oliveira (2019). Subevents detection through topic modeling in social media posts. Future Generation Computer Systems, 93, pp. 290-303.

[7] Mahmud Hasan, Mehmet A Orgun, and Rolf Schwitter (2019). Real-time event detection from the twitter data stream using the Twitternews+ framework. Information Processing \& Management, 56(3), pp. 1146-1165.

[8] Ksenia D Mukhina, Alexander A Visheratin, and Denis Nasonov (2019). Urban events prediction via convolutional neural networks and instagram data. Procedia Computer Science, 156, pp. 176-184.

[9] Linmei Hu, Shuqi Yu, Bin Wu, Chao Shao, and Xiaoli Li (2020). A neural model for joint event detection and prediction. Neurocomputing, 40, pp. 376-384.

[10] Zhenguo Yang, Qing Li, Wenyin Liu, and Jianming Lv (2019). Shared multi-view data representation for multi-domain event detection. IEEE Transactions on Pattern Analysis and Machine Intelligence, 42(5), pp. 1243-1256.

[11] Alomari E., Katib I. and Mehmood, R (2020). Iktishaf: A big data road-traffic event detection tool using Twitter and spark machine learning. Mobile Networks and Applications, pp.1-16.

[12] Wilson S.R., Magdy W., McGillivray B. and Tyson G. (2020). Analyzing temporal relationships between trending terms on twitter and urban dictionary activity. In 12th ACM Conference on Web Science, pp. 155-163.

[13] Ansah J., Liu L., Kang W., Liu J. and Li J. (2020). Leveraging burst in twitter network communities for event detection. World Wide Web, 23(5), pp. 2851-2876.

[14] Halil Kilicoglu and Sabine Bergler (2009). Syntactic dependency based heuristics for biological event extraction. In Proceedings of the BioNLP 2009 Workshop Companion Volume for Shared Task, pages 119-127.

[15] Papadopoulos S., Schinas E., Mezaris V., Troncy R. and Kompatsiaris I.(2013). The 2012 social event detection dataset. In Proceedings of the 4th ACM Multimedia Systems Conference, pp. 102-107.

[16] Thomas Ashish Cherian. (2019). \#CAA tweets till 9/01/2020. The state of Indian politics. 2020-01-09. Retrieved from: https://www.kaggle.com/reach2ashish/caa-tweets-till-9012020.

[17] Wikipedia contributors. (2021). "Citizenship Amendment Act protests." Wikipedia, The Free Encyclopedia. Wikipedia, The Free Encyclopedia, 10 Sep 2021

[18] THE QUINT. (2019). 'Youth Hates Anarchy': PM Modi Addresses Last Mann Ki Baat of 2019. Updated: 29 Dec 2019, 12:28 PM IST. Retrieved from: https://www.thequint.com/news/pm-modi-last-mann-ki-baat-of-2019-december-29-caa-protests.

[19] Nalini Sharma. (2019). 'Citizenship Amendment Act: 60 Petitions To Be Considered By Supreme Court Today'. 18th December, 2019 09:38 IST. Retrieved from:https://www.republicworld.com/india-news/general-news/caa-60-petitions-to-be-considered-bysupreme-court-today.html.

\section{Authors Profile}

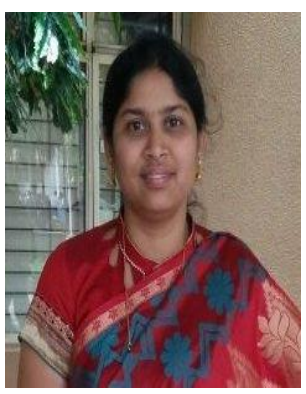

Dr. Jhansi Rani Prathuri, Ph.D. in the field of Computer Science from the University of Hyderabad, India. Working as a Professor at Department of Computer Science and Engineering, CMR Institute of Technology, Bangalore, India. The author also served as head of the department and research center. The author has more than 16 years of teaching experience. Her research interests include Information security and privacy, Cryptography, Big data, Machine learning, Cloud computing, Software engineering, algorithms, and data structures. She has published a lot of research papers at international forums. Her research citations speak about the acceptance and applicability of the research work.

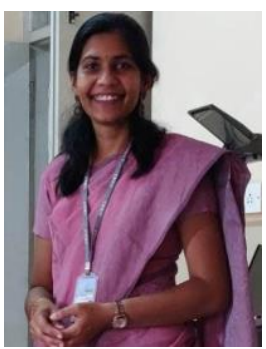

Poonam Tijare, is a research scholar and working as an assistant professor at the Department of Computer Science and Engineering, CMR Institute of Technology, VTU Research center, Bangalore, India. The author has 12 years of experience in the education field. The research interests include Natural language processing, Data mining, Social media analytics, and Machine learning. 